OPEN ACCESS

UWS Academic Portal

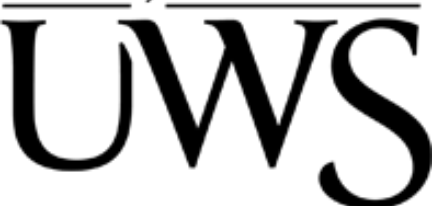

\title{
Vanishing gaps in photonic crystals and other periodic potentials
}

Caffrey, S.; Morozov, G.V.; MacBeath, D.; Sprung, D.W.L.

Published in:

18th International Conference on Transparent Optical Networks (ICTON), 2016

DOI:

10.1109/ICTON.2016.7550309

Published: 25/08/2016

Document Version

Peer reviewed version

Link to publication on the UWS Academic Portal

Citation for published version (APA):

Caffrey, S., Morozov, G. V., MacBeath, D., \& Sprung, D. W. L. (2016). Vanishing gaps in photonic crystals and other periodic potentials. In 18th International Conference on Transparent Optical Networks (ICTON), 2016 [Mo.C6.5] IEEE. https://doi.org/10.1109/ICTON.2016.7550309

\section{General rights}

Copyright and moral rights for the publications made accessible in the UWS Academic Portal are retained by the authors and/or other copyright owners and it is a condition of accessing publications that users recognise and abide by the legal requirements associated with these rights.

Take down policy

If you believe that this document breaches copyright please contact pure@uws.ac.uk providing details, and we will remove access to the work immediately and investigate your claim. 


\title{
Vanishing Gaps in Photonic Crystals and Other Periodic Potentials
}

\author{
S. Caffrey ${ }^{1}$, G. V. Morozov ${ }^{1^{*}}$, D. Macbeath ${ }^{2}$ and D. W. L. Sprung ${ }^{2}$ \\ ${ }^{I}$ Scottish Universities Physics Alliance (SUPA), Institute of Thin Films, Sensors and Imaging, \\ University of the West of Scotland, Paisley PA1 2BE, Scotland, United Kingdom \\ ${ }^{2}$ Department of Physics and Astronomy, McMaster University, Hamilton, ON L8S 4M1, Canada \\ *Tel: (44-141) 848 3676, e-mail: gregory.morozov@uws.ac.uk
}

\begin{abstract}
Using Floquet-Bloch theory, we find incipient bands (vanishing gaps) in the band structures of one-dimensional photonic crystals with a piece-wise constant profile of the refractive index, and in the band structures of continuously differentiable periodic potentials. The emphasis is on potentials that allow solutions in an exact analytical form.
\end{abstract}

Keywords: Floquet-Bloch theory, band structure of periodic potentials, vanishing gaps (incipient bands).

\section{INTRODUCTION}

The mathematical description of many physical problems, involving periodic variations either in space or time, reduces to Hill's differential equation in the form

$$
\frac{d^{2} \Psi(z)}{d z^{2}}+Q(z) \Psi(z)=0, \quad Q(z)=Q(z+d) .
$$

This includes, for example, light propagation in a one-dimensional (1D) photonic crystal, or the motion of electrons in a $1 \mathrm{D}$ superlattice.

According to Floquet-Bloch theory, see [1-3], among the fundamental systems of solutions of equation (1) there is a special one called the Floquet-Bloch system. This special fundamental system typically consists of two particular solutions $F_{1,2}(z)$, called Bloch waves, with the property under translation

$$
F_{1,2}(z+d)=\rho_{1,2} F_{1,2}(z),
$$

where the constants $\rho_{1,2}$ are non-zero, generally complex-valued numbers, called Floquet multipliers. They obey the relation

$$
\rho_{1} \rho_{2}=1,
$$

In the allowed bands (regions of stability) both Bloch waves are bounded propagating functions. In the bandgaps (regions of instability) one Bloch wave decreases along the axis of propagation, while the other grows exponentially. That growing wave is an unphysical solution in case of an infinite periodic structure. Accordingly, in the bandgaps the multipliers $\rho_{1,2}$ become real-valued numbers.

On the boundaries (bandedges) between allowed bands and bandgaps, the two Bloch waves coincide, i.e. $F_{2}(z)=F_{1}(z) \equiv F(z)$. The constants $\rho_{1,2}$ also coincide, i.e. $\rho_{1}=\rho_{2} \equiv \rho$ and, in accordance with equation (3), either $\rho=1$ or $\rho=-1$. In the former case the function $F(z)$ has a period $d$ while in the latter case it has a period $2 d$. Then, to complete the fundamental system of equation (1) another particular solution is required. That particular solution is often taken in the form of a hybrid Floquet mode, with the property

$$
G(z+d)=\rho G(z)+\rho d G(z) .
$$

In practice, Bloch waves, hybrid Floquet modes, Floquet multipliers are all expressed in terms of normalized solutions $u(z)\left[u(0)=1, u^{\prime}(0)=0\right]$ and $v(z)\left[v(0)=0, v^{\prime}(0)=1\right]$. The structure of allowed bands and bandgaps is defined by the dispersion equation

$$
\cos (\mu d)=\left[u(d)+v^{\prime}(d)\right] / 2,
$$

where $\mu$ is the Bloch wave number related to the Floquet multipliers by $\rho_{1,2}=\exp ( \pm i \mu d)$. One can see that in the allowed bands $-1<\cos (\mu d)<1$, in the bandgaps $|\cos (\mu d)|>1$, and at the bandedges $\cos (\mu d)= \pm 1$.

In the spectrum of Floquet-Bloch solutions, a case of special interest is so-called incipient bands (or vanishing gaps). Those are points of contact between distinct allowed bands (i.e. a special type of band crossing). Mathematically, in that case $\rho_{1}=\rho_{2} \equiv \rho= \pm 1$, but the two Bloch waves are independent periodic functions, with 
the same periodicity ( $d$ if $\rho=1$, or $2 d$ if $\rho=-1$ ), accordingly at those points $\cos (\mu d)= \pm 1$ and, in addition, $v(d)=0, u^{\prime}(d)=0$.

It is known that vanishing gaps do not occur for the Dirac comb, nor the Mathieu potential, nor for a sawtooth potential $[4,5]$. In this paper we summarize our recent findings on certain periodic potentials for which incipient bands do exist. This includes not only a well-known case of a binary photonic crystal (analogue of the KronigPenney model in quantum mechanics) but also a small set of periodic potentials having a continuouslydifferentiable function $Q(z)$ in equation (1).

\section{VANISHING GAPS IN BINARY PHOTONIC CRYSTALS}

In this section, we illustrate existence of incipient bands in a spectrum of light impinging normally on a binary crystal, illustrated in Fig. 1, for the case $d_{1}=d_{2}=d / 2$ (basis layers of the same thickness). The dispersion equation (5) takes the form

$$
\cos (\mu d)=\frac{\cos \left(k n_{\mathrm{av}} d\right)-r_{21}^{2} \cos \left(r_{21} k n_{\mathrm{av}} d\right)}{1-r_{21}^{2}},
$$

where $k=2 \pi / \lambda$ is the vacuum wavenumber of impinging light and $r_{21}=\left(n_{2}-n_{1}\right) /\left(n_{2}+n_{1}\right)$ is the amplitude Fresnel reflection coefficient on the boundary between the layers $n_{2}$ and $n_{1}$. The results are shown in Fig. 2. The incipient bands are a set of points defined by either

$$
\begin{cases}k n_{\mathrm{av}} d=2 m \pi, & m=2,3, \ldots, \\ r_{21}= \pm l / m, & l=1, \ldots, m-1,\end{cases}
$$

where the Bloch waves have periodicity $d$, or by

$$
\begin{cases}k n_{\mathrm{av}} d=(2 m-1) \pi, & m=2,3, \ldots, \\ r_{21}= \pm(2 l-1) /(2 m-1), & l=1, \ldots, m-1,\end{cases}
$$

where the Bloch waves have periodicity $2 d$.

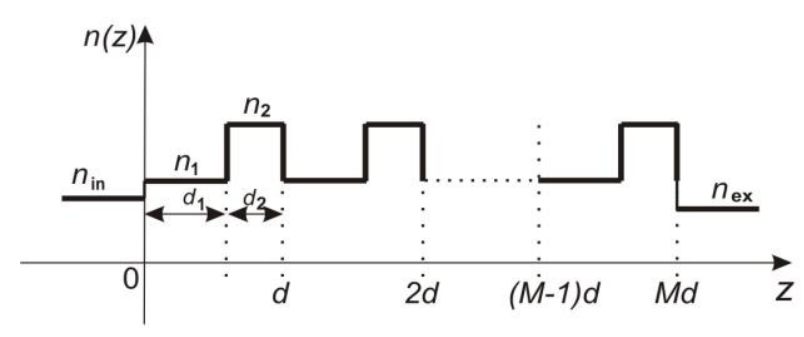

Fig. 1. Binary photonic crystal with the layers of refractive indices $n_{1}, n_{2}$ and thicknesses $d_{1}, d_{2}$, $M$ is the number of periods.

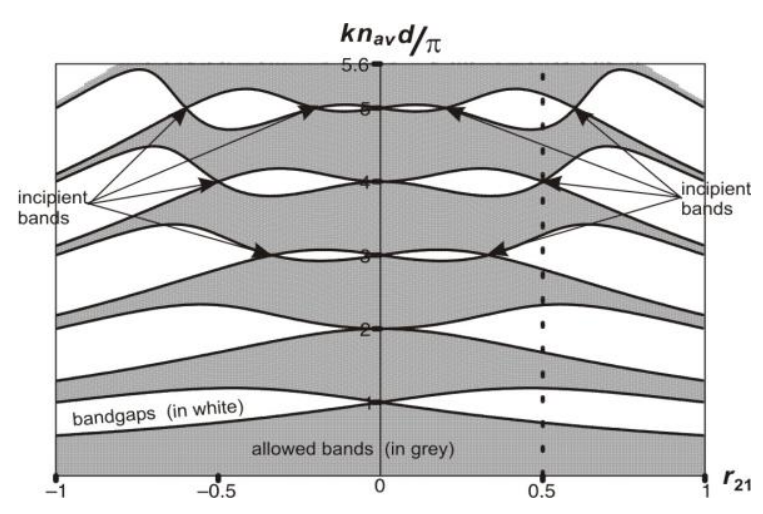

Fig. 2. Band structure of a binary crystal for normal incidence for the case $d_{1}=d_{2}=d / 2$, $r_{21}=\left(n_{2}-n_{1}\right) /\left(n_{2}+n_{1}\right), n_{\mathrm{av}}=\left(n_{2} d_{2}+n_{1} d_{1}\right) / d$.

The above problem about light normally incident on a binary photonic crystal with layers of equal geometrical thicknesses $\left(d_{1}=d_{2}\right)$ is just one example of incipient bands (vanishing gaps) in the band structure of piece-wise constant periodic potentials. Other confirmed examples where incipient bands occur, include normal incidence of light on a binary crystal with layers of equal optical thicknesses $\left(n_{1} d_{1}=n_{2} d_{2}\right)$, see [6], oblique incidence of light on an arbitrary binary crystal, see [7], etc. We should note that in all those problems the incipient bands (vanishing gaps) appear in the form of a discrete set of points.

\section{VANISHING GAPS IN CONTINUOUS PERIODIC POTENTIALS}

In this section we illustrate existence of incipient bands for three periodic potentials with continuouslydifferentiable functions $Q(z)$. The first example is the Casperson potential [8,9], see Fig. 3, given by

$$
Q_{c}=\frac{a}{(1+q \cos 2 z)^{4}}+\frac{4 q \cos (2 z)}{1+q \cos 2 z}, \quad-1<q<1 .
$$


For this potential the dispersion equation (5) takes the form

$$
\cos (\mu d)=\cos \left[\pi \sqrt{\frac{a}{\left(1-q^{2}\right)^{3}}}\right],
$$

where $d=\pi$ is the period of the potential. The band structure is shown in Fig. 4. There is only one allowed band $(a>0)$ separated by a bandedge $(a=0)$ from a single bandgap $(a<0)$. The incipient bands are curves defined by

$$
\frac{a}{\left(1-q^{2}\right)^{3}}=m^{2}, \quad m=1,2,3,4, \ldots,
$$

where the Bloch waves have periodicity $d=\pi$ for $m=2,4, \ldots$, and $2 d=2 \pi$ for $m=1,3, \ldots$

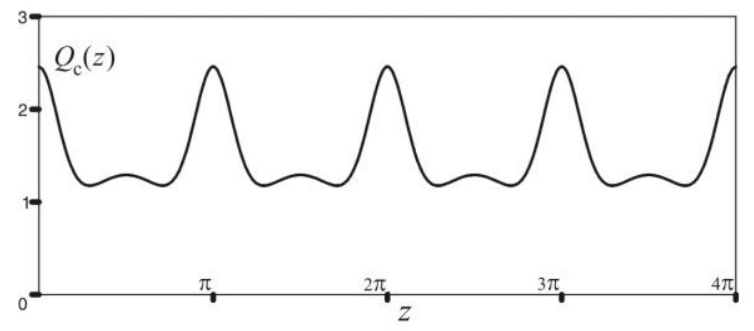

Fig. 3. Casperson potential for $q=-0.25, a=1.20$.

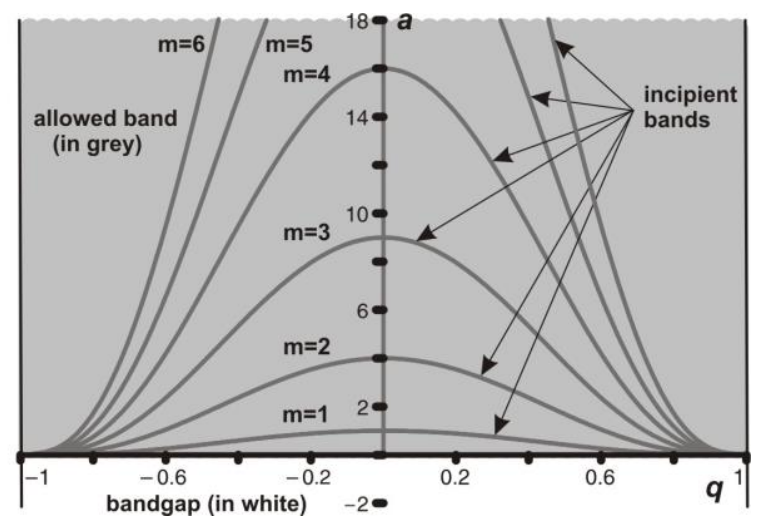

Fig. 4. Band structure of the Casperson potential, only the first six incipient bands are shown.

The next example is the Wu potential $[9,10]$, see Fig. 5, which is somewhat similar to the Casperson one. It is given by the function

$$
Q_{w}=\frac{a+q^{2} \sin ^{2} 2 z}{(1+q \cos 2 z)^{2}}+\frac{2 q \cos (2 z)}{1+q \cos 2 z}, \quad-1<q<1,
$$

with the dispersion equation (5) taking the form

$$
\cos (\mu d)=\cos \left[\pi \sqrt{\frac{a}{1-q^{2}}}\right] .
$$

The band structure is shown in Fig. 6. Similar to the Casperson potential, there is a single allowed band $(a>0)$ separated by a bandedge $(a=0)$ from a single bandgap $(a<0)$. The incipient bands are curves defined by

$$
\frac{a}{1-q^{2}}=m^{2}, \quad m=1,2,3,4, \ldots,
$$

where the Bloch waves have periodicity $d=\pi$ for $m=2,4, \ldots$, and $2 d=2 \pi$ for $m=1,3, \ldots$.

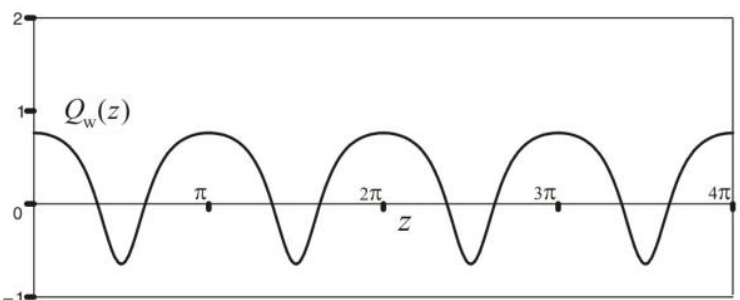

Fig. 5. Wu potential for $q=0.45, a=0.30$.

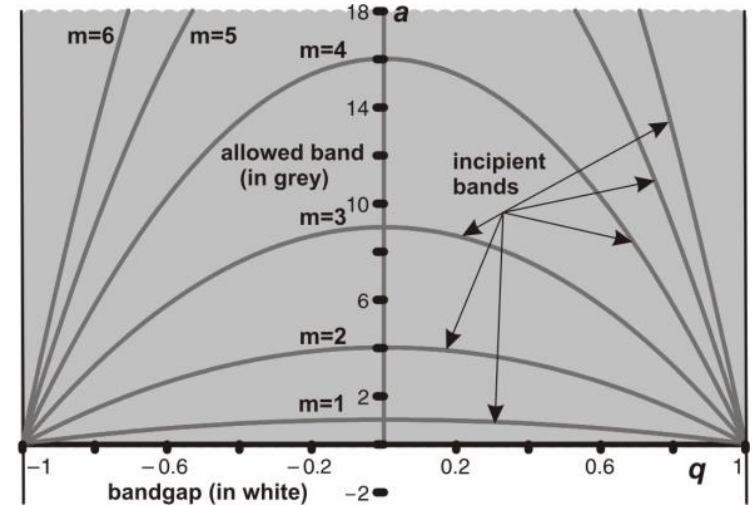

Fig. 6. Band structure of the Wu potential, only the first six incipient bands are shown. 
The final example is the Takayama potential $[11,12]$, see Fig. 7, given by the function

$$
Q_{t}=1+\frac{a\left(1-q^{2}\right)}{(1+q \cos 2 z)^{2}}, \quad-1<q<1,
$$

with the dispersion equation (5)

$$
\cos (\mu d)=\cos [\pi \sqrt{a+1}]
$$

The band structure is shown in Fig. 8. An allowed band $(a>-1)$ is separated by a bandedge $(a=-1)$ from a single bandgap $(a<-1)$. The incipient bands are straight lines defined by

$$
a=m^{2}-1, \quad m=1,2,3,4, \ldots,
$$

where the Bloch waves have periodicity $d=\pi$ for $m=2,4, \ldots$, and $2 d=2 \pi$ for $m=1,3, \ldots$

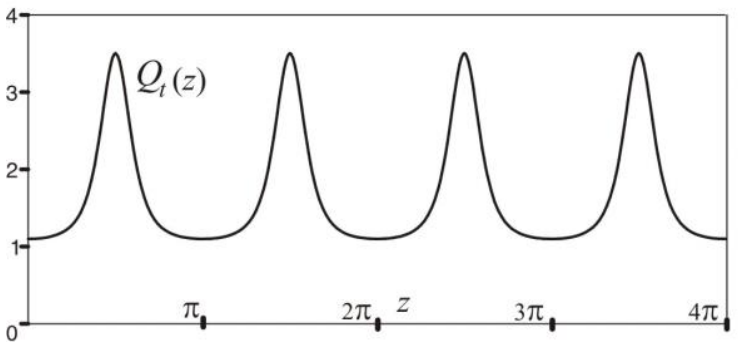

Fig. 7. Takayama potential for $q=2 / 3, a=1 / 2$.

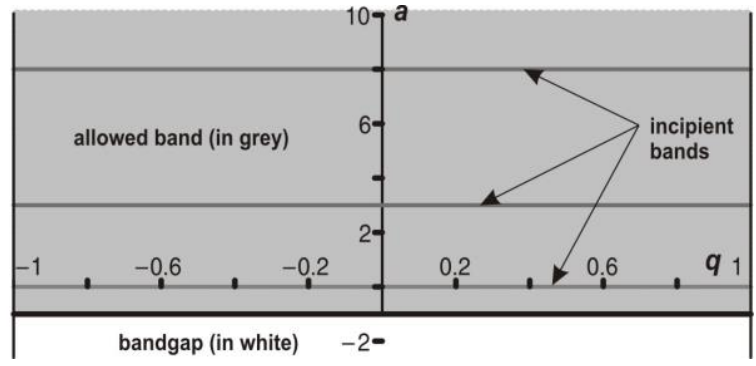

Fig. 8. Band structure of the Takayama potential, only the first three incipient bands are shown.

The above three continuous periodic potentials add to the set of exactly solvable Hill's equations. The band structure of each potential features a single bandgap separated by a bandedge from a single allowed band, which is divided into pieces by incipient bands (vanishing gaps). Those incipient bands are a set of continuous curves, rather than a set of points as was observed for the piece-wise constant periodic potential.

\section{CONCLUSIONS}

We analysed the band structures of certain exactly solvable Hill's equations with both piece-wise constant and continuously-differentiable periodic potentials. Particular attention was given to the incipient bands (vanishing gaps) where all solutions of Hill's equation become periodic functions. A further logical step would be to obtain an analytical criterion for the existence of incipient bands in an arbitrary periodic potential.

\section{ACKNOWLEDGEMENTS}

We are grateful to NCERC Canada for continuing support under Discovery Grant RGPIN-3198 (DMB, DWLS).

\section{REFERENCES}

[1] W. Magnus, S. Winkler: Hill's Equation, Dover, New York, 2004.

[2] M. S. P. Eastham: The Spectral Theory of Periodic Differential Equations, Scottish Academic Press, Edinburgh, 1975.

[3] J. J. Stoker: Nonlinear Vibrations, Waverly Press, New York, 1950.

[4] G. V. Morozov, D. W. L. Sprung, J. Martorell: One-dimensional photonic crystals with a sawtooth refractive index: another exactly solvable potential, New Journal of Physics, vol. 15, 103009, Oct. 2013.

[5] S. Caffrey, G. V. Morozov, D. W. L. Sprung, J. Martorell: Band structure analysis of binary and sawtooth photonic crystals by Floquet-Bloch method, Optical and Quantum Electronics, to be submitted.

[6] G. V. Morozov, D. W. L. Sprung: Floquet-Bloch Waves in One-Dimensional Photonic Crystals, EPL (Europhysics Letters), vol. 96, 54005, Dec. 2011.

[7] G. V. Morozov, F. Placido, D. W. L. Sprung: Light scattering from one-dimensional photonic crystals under total internal reflection, in Proc. ICTON 2013, Cartagena, Spain, July 2013, paper WeC6.

[8] L. W. Casperson: Solvable Hill equation, Phys. Rev. A, vol. 30, pp. 2749-2751, Nov. 1984.

[9] S. Caffrey, G. V. Morozov, D. MacBeath, D. W. L. Sprung: Floquet-Bloch analysis of analytically solvable Hill equations with continuous potentials, JOSA B, vol. 33, pp. 1190-1196, June 2016.

[10] S-M. Wu, C-C. Shih: Construction of solvable Hill equations, Phys. Rev. A, vol. 32, pp. 3736-3738, Dec. 1985.

[11] K. Takayama: Note on solvable Hill equations, Phys. Rev. A, vol. 34, pp. 4408-4410, Nov. 1986.

[12] G. V. Morozov, D. W. L. Sprung: Band structure analysis of an analytically solvable Hill equation with continuous potential, J. Opt., vol. 17, 035607, Feb. 2015. 